\title{
ON THE STRONG SUMMABILITY OF FOURIER SERIES
}

\section{GEN-ICHIRÔ SUNOUCHI}

This paper consists of three parts. In the first part we complete one of the deepest theorems due to Littlewood and Paley $[3,4] .{ }^{1}$ Our theorem was already proved by Marcinkiewicz and Zygmund [4]. But the proof given here is very simple and direct. In the second part a strong summability theorem is proved. It is the completion of our former theorem [5]. We use the method due to Zygmund [8] for the proof. Finally we prove a strong summability theorem concerning lacunary sequences of partial sums (Zalcwasser [6]).

1. Let $f(\theta)$ be an integrable function with period $2 \pi$ and its Fourier series be $f(\theta) \sim \sum_{n=1}^{\infty}\left(a_{n} \cos n \theta+b_{n} \sin n \theta\right)$, assuming $a_{0}=0$ for the sake of simplicity. If we put $z=\rho e^{i \theta}$ and

$$
\phi(z)=\sum_{n=1}^{\infty}\left(a_{n}-i b_{n}\right) z^{n},
$$

then $f(\theta)$ becomes the boundary function of the harmonic function $\Re \phi(z)$. Let us denote by $p, q$, and $r$ real numbers satisfying

$$
r>1, \quad 1<p \leqq 2 \leqq q<\infty,
$$

and by $A, B, \cdots$ absolute constants, not always the same from one occurrence to another.

THEOREM 1. Let $f(\theta)$ be a function of the class $L^{r}$, then

$$
\begin{aligned}
A \int_{0}^{2 \pi}\left|\phi\left(e^{i \theta}\right)\right| r d \theta & \leqq \int_{0}^{2 \pi}\left\{\int_{0}^{1}(1-\rho)\left|\phi^{\prime}(z)\right|^{2} d \rho\right\}^{r / 2} d \theta \\
& \leqq B \int_{0}^{2 \pi}\left|\phi\left(e^{i \theta}\right)\right|^{r} d \theta,
\end{aligned}
$$

$$
\begin{aligned}
& \int_{0}^{2 \pi}\left\{\int_{0}^{1}(1-\rho)^{q-1}\left|\phi^{\prime}(z)\right|^{q} d \rho\right\}^{r / q} d \theta \leqq C \int_{0}^{2 \pi}\left|\phi\left(e^{i \theta}\right)\right|^{r} d \theta \\
& D \int_{0}^{2 \pi}\left|\phi\left(e^{i \theta}\right)\right|^{r} d \theta \leqq \int_{0}^{2 \pi}\left\{\int_{0}^{1}(1-\rho)^{p-1}\left|\phi^{\prime}(z)\right|^{p} d \rho\right\}^{r / p} d \theta
\end{aligned}
$$

(1.1) is due to Littlewood and Paley [4]. In order to prove (1.2) we put

Received by the editors October 18, 1948 and, in revised form, May 14, 1949.

1 Numbers in brackets refer to the bibliography at the end of the paper. 


$$
\Phi(\theta)=\max (|\phi(z)| ; z \in S(\theta))
$$

where $S(\theta)$ is a kite-shaped region. ${ }^{2}$ Then $\left|\phi^{\prime}\right| \leqq A \Phi /(1-\rho)$. If we put

$$
g(\theta)=\left(\int_{0}^{1}(1-\rho)\left|\phi^{\prime}(z)\right|^{2} d \rho\right)^{1 / 2},
$$

then we have

$$
\begin{aligned}
\left\{\int_{0}^{2 \pi}\right. & \left.\left(\int_{0}^{1}(1-\rho)^{q-1}\left|\phi^{\prime}\right| q d \rho\right)^{r / q} d \theta\right\}^{1 / r} \\
& \leqq\left\{\int_{0}^{2 \pi}\left(\int_{0}^{1}\left|\phi^{\prime}\right|^{2}(1-\rho) A \Phi^{q-2} d \rho\right)^{r / q} d \theta\right\}^{1 / r} \\
& \leqq\left\{\int_{0}^{2 \pi}\left(A \Phi^{q-2} \cdot g^{2}\right)^{r / q} d \theta\right\}^{1 / r} \\
& \leqq\left(B \int_{0}^{2 \pi} \Phi^{(1-2 / q) r} g^{2 r / q} d \theta\right)^{1 / r} .
\end{aligned}
$$

Since $0<2 / q<1$, if we apply Hölder's inequality, the right-hand side becomes less than

$$
\begin{aligned}
C\left\{\left(\int_{0}^{2 \pi} \Phi^{r} d \theta\right)^{1-2 / q}\right. & \left.\left(\int_{0}^{2 \pi}|g|^{r} d \theta\right)^{2 / q}\right\}^{1 / r} \\
& \leqq C\left\{\left(\int_{0}^{2 \pi} \Phi^{r} d \theta\right)^{1 / r}\right\}^{1-2 / q} \cdot\left\{\left(\int_{0}^{2 \pi}|g|^{r} d \theta\right)^{1 / r}\right\}^{2 / q} .
\end{aligned}
$$

By the maximal theorem due to Hardy and Littlewood the last term is less than

$$
\begin{aligned}
D\left\{\left(\int_{0}^{2 \pi}|\phi| r d \theta\right)^{1 / r}\right\}^{1-2 / Q} \cdot\left\{\left(\int_{0}^{2 \pi}|g|^{r} d \theta\right)^{1 / r}\right\}^{2 / Q} & \\
& \leqq E\left(\int_{0}^{2 \pi}|\phi| r d \theta\right)^{1 / r} .
\end{aligned}
$$

Thus we get (1.2) by (1.1).

For the proof of (1.3) we put

$$
h(\theta)=\left(\int_{0}^{1}(1-\rho)^{p-1}\left|\phi^{\prime}(z)\right|^{p} d \rho\right)^{1 / p} .
$$

2 See Littlewood and Paley [4]. 
528

G. I. SUNOUCHI

[August

Then we have

$$
\begin{aligned}
& \left\{\int_{0}^{2 \pi}\left(\int_{0}^{1}(1-\rho)\left|\phi^{\prime}(z)\right|^{2} d \rho\right)^{r / 2} d \theta\right\}^{1 / r} \\
& \leqq\left\{\int_{0}^{2 \pi}\left(\int_{0}^{1}\left|\phi^{\prime}\right| p(1-\rho)^{p-1} A \Phi^{2-p} d \rho\right)^{r / 2} d \theta\right\}^{1 / r} \\
& \leqq\left\{\int_{0}^{2 \pi}\left(A \Phi^{2-p}|h(\theta)|^{p}\right)^{r / 2} d \theta\right\}^{1 / r} \leqq B\left\{\int_{0}^{2 \pi} \Phi^{(1-p / 2) r} \cdot h^{p r / 2} d \theta\right\}^{1 / r} \\
& \leqq C\left\{\left(\int_{0}^{2 \pi} \Phi^{r} d \theta\right)^{1-p / 2}\left(\int_{0}^{2 \pi} h^{r} d \theta\right)^{p / 2}\right\}^{1 / r} \\
& \leqq C\left\{\left(\int_{0}^{2 \pi} \Phi^{r} d \theta\right)^{1 / r}\right\}^{1-p / 2}\left\{\left(\int_{0}^{2 \pi} h^{r} d \theta\right)^{1 / r}\right\}^{p / 2} \\
& \leqq D\left\{\left(\int_{0}^{2 \pi}|\phi|^{r} d \theta\right)^{1 / r}\right\}^{1-p / 2}\left\{\left(\int_{0}^{2 \pi} h^{r} d \theta\right)^{1 / r}\right\}^{p / 2} .
\end{aligned}
$$

Thus we have

$$
\left(\int_{0}^{2 \pi} g^{r} d \theta\right)^{1 / r} \leqq E\left(\int_{0}^{2 \pi} h^{r} d \theta\right)^{1 / r}
$$

Hence by (1.1) we get (1.3).

2. THEOREM 2. Let $s_{n}\left(e^{i \theta}\right)$ and $\tau_{n}\left(e^{i \theta}\right)$ denote the partial sums and the arithmetic mean of Fourier power series of $\phi\left(e^{i \theta}\right)$ belonging to the class $H^{r}$, respectively. Then we have

$$
\begin{aligned}
A \int_{0}^{2 \pi}\left|\phi\left(e^{i \theta}\right)\right|^{r} d \theta & \leqq \int_{0}^{2 \pi}\left(\sum_{n=1}^{\infty}\left|s_{n}\left(e^{i \theta}\right)-\tau_{n}\left(e^{i \theta}\right)\right|^{2} / n\right)^{r / 2} d \theta \\
& \leqq B \int_{0}^{2 \pi}\left|\phi\left(e^{i \theta}\right)\right|^{r} d \theta
\end{aligned}
$$

$$
\int_{0}^{2 \pi}\left(\sum_{n=1}^{\infty} \frac{1}{n}\left|s_{n}\left(e^{i \theta}\right)-\tau_{n}\left(e^{i \theta}\right)\right| q\right)^{r / Q} d \theta \leqq C \int_{0}^{2 \pi}\left|\phi\left(e^{i \theta}\right)\right| r d \theta
$$

(2.3) $D \int_{0}^{2 \pi}\left|\phi\left(e^{i \theta}\right)\right| r d \theta \leqq \int_{0}^{2 \pi}\left(\sum_{n=1}^{\infty} \frac{1}{n}\left|s_{n}\left(e^{i \theta}\right)-\tau_{n}\left(e^{i \theta}\right)\right|^{p}\right)^{r / p} d \theta$.

(2.1) is due to Zygmund [8, 9], and (2.2) is given in my former paper [5]. For the proof of the theorem we require the following lemmas. 
Lemma 1. Let $f_{1}, f_{2}, \cdots$ be a sequence of integrable functions and let $s_{n, v}$ denote the $\nu$ th partial sum of the Fourier series of $f_{n}$. Then

$$
\int_{0}^{2 \pi}\left(\sum_{n=1}^{\infty}\left|s_{n, k_{n}}(\theta)\right|^{m}\right)^{r / m} d \theta \leqq A \int_{0}^{2 \pi}\left(\sum_{n=1}^{\infty}\left|f_{n}\right|^{m}\right)^{r / m} d \theta,
$$

where $r>1$ and $m>1$.

The inequality is also valid for conjugate series.

This lemma is due to Boas and Bochner [1].

If we write

$$
\begin{aligned}
& s_{\nu}(\rho, \theta)=\sum_{n=1}^{\infty}\left(a_{n} \cos n \theta+b_{n} \sin n \theta\right) \rho^{n}, \\
& f(\rho, \theta)=\sum_{n=1}^{\infty}\left(a_{n} \cos n \theta+b_{n} \sin n \theta\right) \rho^{n},
\end{aligned}
$$

then we have the following lemma.

LEMMA 2. Let $s_{n, v}(\rho, \theta)$ denote the sum derived from $f_{n}(\theta)$ similarly as $s_{v}(\rho, \theta)$ from $f(\theta)$. Then under the hypothesis of Lemma 1 we have

$$
\int_{0}^{2 \pi}\left(\sum_{n=1}^{\infty}\left|s_{n, k_{n}}\left(\rho_{n}, \theta\right)\right|^{m}\right)^{2 / m} d \theta \leqq A \int_{0}^{2 \pi}\left(\sum_{n=1}^{\infty}\left|f_{n}(\theta)\right|^{m}\right)^{r / m} d \theta,
$$

where $0 \leqq \rho_{n} \leqq 1$. The sums $s_{n,}$ may be replaced by the conjugate ones.

This lemma is easily derived from Lemma 1 by Zygmund's argument by a slight modification.

LEMMA 3. Let $0 \leqq \rho_{n}<1$ and $\Delta_{n}$ denote an arbitrary interval situated in $\left(\rho_{n}, 1\right)$. Then under the hypothesis of Lemma 1 we have

$$
\begin{aligned}
\int_{0}^{2 \pi}\left(\sum_{n=1}^{\infty}\left|s_{n, k_{n}}\left(\rho_{n}, \theta\right)\right|^{m}\right)^{r / m} d \theta & \\
& \leqq A \int_{0}^{2 \pi}\left(\sum_{n=1}^{\infty} \frac{1}{\Delta_{n}} \int_{\Delta_{n}}\left|f_{n}(\rho, \theta)\right|^{m} d \rho\right)^{r / m} d \theta .
\end{aligned}
$$

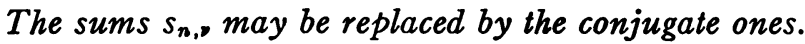

This is immediate from Lemma 2.

Proof of (2.2). We have

$$
s_{n}\left(e^{i \theta}\right)-\tau_{n}\left(e^{i \theta}\right)=-i s_{n}^{\prime}\left(e^{i \theta}\right) /(n+1) .
$$


By Abel's transformation

$$
s_{n}^{\prime}\left(e^{i \theta}\right)=\rho^{-n} s_{n}^{\prime}\left(\rho e^{i \theta}\right)-(1-\rho) \sum_{\nu=0}^{n-1} \rho^{-r-1} s_{\nu}^{\prime}\left(\rho e^{i \theta}\right) .
$$

Therefore

$$
\begin{aligned}
\left|s_{n}^{\prime}\left(e^{i \theta}\right)\right|^{q} & \leqq A\left\{\rho^{-q n}\left|s_{n}^{\prime}\left(\rho e^{i \theta}\right)\right|^{q}+(1-\rho)^{q}\left(\sum_{\nu=0}^{n-1} \rho^{-r-1}\left|s_{\nu}^{\prime}\left(\rho e^{i \theta}\right)\right|\right)^{q}\right\} \\
& \leqq B\left\{\rho^{-q n}\left|s_{n}^{\prime}\left(\rho e^{i \theta}\right)\right|^{q}+\frac{1-\rho}{\rho^{(q-1) n}} \sum_{\nu=0}^{n-1} \rho^{-\nu-1}\left|s_{\nu}^{\prime}\left(\rho e^{i \theta}\right)\right| q\right\} .
\end{aligned}
$$

Let $\rho_{n}=1-1 /(n+1)$ and $\Delta_{n}=\left(\rho_{n}, \rho_{n+1}\right)$ and let $I$ be the left-hand side integral of (2.2). Then we have

$$
\begin{aligned}
I & \leqq C \int_{0}^{2 \pi}\left(\sum_{n=1}^{\infty} \frac{\left|s_{n}^{\prime}\left(\rho_{n} e^{i \theta}\right)\right|^{q}}{n^{q+1} \rho_{n}^{q n}}\right. \\
& \left.\quad+\sum_{n=1}^{\infty} \frac{1-\rho_{n}}{n^{q+1} \rho_{n}^{(q-1) n}} \sum_{n=0}^{n-1} \rho_{n}^{-r-1}\left|s_{\nu}^{\prime}\left(\rho_{n} e^{i \theta}\right)\right|^{q}\right)^{r / q} d \theta \\
& \leqq D \int_{0}^{2 \pi}\left(\sum_{n=1}^{\infty} \frac{\left|s_{n}^{\prime}\left(\rho_{n} e^{i \theta}\right)\right|^{q}}{n^{q+1} \Delta_{n}} \int_{\Delta_{n}}\left|\phi^{\prime}\left(\rho e^{i \theta}\right)\right|^{q} d \rho\right. \\
& \left.+\sum_{n=1}^{\infty} \frac{1}{n^{q+2}} \sum_{n=0}^{n-1} \frac{1}{\Delta_{n}} \int_{\Delta_{n}}\left|\phi^{\prime}\left(\rho e^{i \theta}\right)\right|^{q} d \rho\right)^{r / q} d \theta \\
& \leqq E \int_{0}^{2 \pi}\left(\sum_{n=1}^{\infty} \frac{n^{2} \cdot n^{q-1}}{n^{q+1}} \int_{\Delta_{n}}(1-\rho)^{q-1}\left|\phi^{\prime}\left(\rho e^{i \theta}\right)\right|^{q} d \rho\right)^{r / q} d \theta \\
& \leqq F \int_{0}^{2 \pi}\left(\int_{0}^{1}(1-\rho)^{q-1}\left|\phi^{\prime}\left(\rho e^{i \theta}\right)\right|^{q} d \rho\right)^{r / q} d \theta \\
& \leqq G \int_{0}^{2 \pi}\left|\phi\left(e^{i \theta}\right)\right|^{r} d \theta
\end{aligned}
$$

from (1.2). Thus we obtain (2.2).

Proof of (2.3). We may write

$$
\left|\phi^{\prime}\left(\rho e^{i \theta}\right)\right|=\left|\sum_{\nu=1}^{\infty} \nu c_{\nu} \rho^{\nu-1} e^{i v \theta}\right|=(1-\rho)\left|\sum_{\nu=1}^{\infty} s_{\nu}^{\prime} \rho^{p-1}\right| .
$$

If we put $\rho_{n}=1-1 / n$, then we have 


$$
\begin{aligned}
\int_{0}^{1} & (1-\rho)^{p-1}\left|\phi^{\prime}\right| p d \rho=\sum_{n=1}^{\infty} \int_{\rho_{n}}^{\rho_{n+1}}(1-\rho)^{p-1}\left|\phi^{\prime}\right|^{p} d \rho \\
& \leqq \sum_{n=1}^{\infty} \int_{p_{n}}^{p_{n+1}}(1-\rho)^{p-1}\left[(1-\rho) \sum_{\nu=1}^{\infty}\left|s_{\nu}^{\prime}\right| \rho^{\nu-1}\right]^{p} d \rho \\
& \leqq \sum_{n=1}^{\infty} \frac{1}{n^{p+1}}\left[\left(1-\rho_{n}\right) \sum_{\nu=1}^{\infty}\left|s_{\nu}^{\prime}\right| \rho_{n+1}^{\nu-1}\right]^{p} \\
& \leqq A \sum_{n=1}^{\infty} \frac{1}{n^{2 p+1}}\left(\sum_{\nu=1}^{\infty}\left|s_{\nu}^{\prime}\right| \rho_{n+1}^{p-1}\right)^{p} \\
& \leqq B \sum_{n=1}^{\infty} \frac{1}{n^{2 p+1}}\left(\sum_{\nu=1}^{n}\left|s_{\nu}^{\prime}\right| \rho_{n+1}^{\nu-1}\right)^{p}+B \sum_{n=1}^{\infty} \frac{1}{n^{2 p+1}}\left(\sum_{\nu=n+1}^{\infty}\left|s_{\nu}^{\prime}\right| \rho_{n+1}^{\nu-1}\right)^{p} \\
& =P+Q
\end{aligned}
$$

say. We have

$$
\begin{aligned}
P & \leqq \sum_{n=1}^{\infty} \frac{1}{n^{2 p+1}}\left(\sum_{\nu=1}^{n}\left|s_{\nu}^{\prime}\right|^{p}\right)\left(\sum_{\nu=1}^{n} \rho_{n+1}^{p^{\prime}(\nu-1)}\right)^{p / p^{\prime}} \\
& \leqq C \sum_{n=1}^{\infty} \frac{1}{n^{2 p+1}}\left(\sum_{\nu=1}^{n}\left|s_{\nu}^{\prime}\right|^{p}\right) \cdot n^{p / p^{\prime}} \\
& \leqq C \sum_{n=1}^{\infty} \frac{1}{n^{p+2}} \sum_{\nu=1}^{n}\left|s_{\nu}^{\prime}\right|^{p} \leqq C \sum_{\nu=1}^{\infty} \frac{\left|s_{\nu}^{\prime}\right|^{p}}{\nu^{p+1}}
\end{aligned}
$$

where $1 / p+1 / p^{\prime}=1$, and

$$
\begin{aligned}
Q & \leqq \sum_{n=1}^{\infty} \frac{1}{n^{2 p+1}}\left(\sum_{\nu=n+1}^{\infty}\left|s_{\nu}^{\prime}\right| \begin{array}{c}
\nu-1 \\
\rho_{n+1}
\end{array}\right)^{p} \\
& \leqq D \sum_{n=1}^{\infty} \frac{1}{n^{2 p+1}}\left(\sum_{\nu=n+1}^{\infty} \frac{\left|s_{\nu}^{\prime}\right|^{p}}{\nu^{p+2}}\right)\left(\sum_{\nu=n+1}^{\infty} \nu^{(1+2 / p) p^{\prime}} \begin{array}{c}
p^{\prime}(\nu-1) \\
\rho_{n+1}
\end{array}\right)^{p / p^{\prime}} \\
& \leqq D \sum_{n=1}^{\infty} \frac{1}{n^{2 p+1}}\left(\sum_{v=n+1}^{\infty} \frac{\left|s_{\nu}^{\prime}\right|^{p}}{\nu^{p+2}}\right)\left(\sum_{\nu=n+1}^{\infty} \cdot \nu^{3 p^{\prime}-2} \rho_{n+1}^{p^{\prime}(\nu-1)}\right)^{p / p^{\prime}} \\
& \leqq D \sum_{n=1}^{\infty} \sum_{\nu=n+1}^{\infty}\left|s_{\nu}^{\prime}\right| p / \nu^{p+2} \leqq E \sum_{\nu=1}^{\infty}\left|s_{\nu}^{\prime}\right|^{p / \nu^{p+1}} .
\end{aligned}
$$

Since $\left|s_{\nu}^{\prime}\right| /(\nu+1)=\left|s_{\nu}-\tau_{\nu}\right|$, we get (2.2) by (1.2).

3. TheOREM 3. Let $f(\theta)$ be a function belonging to $L^{r}(r>1)$, and $\left\{p_{n}\right\}$ and $\left\{d_{n}\right\}$ be arbitrary increasing sequences of positive integers such that

$$
p_{n} / d_{n}=O\left(p_{n+1}-p_{n}\right) .
$$


Then we have

$$
\int_{0}^{2 \pi}\left(\sum_{\nu=1}^{\infty}\left|s_{p_{\nu}}-\tau_{p_{\nu}}\right| q / d_{\nu}\right)^{r / q} d \theta \leqq A \int_{0}^{2 \pi}|f(\theta)|^{r} d \theta(q \geqq 2) .
$$

Especially if

$$
p_{n} / n=O\left(p_{n+1}-p_{n}\right)
$$

then

$$
\sum_{\nu=1}^{\infty}\left|s_{p_{\nu}}-f\right|^{m} / \nu<\infty \quad(m \geqq 1)
$$

for almost all $\theta$.

The proof of (3.1) runs similarly to that of (2.2). In this case we have to replace $\Delta_{n}$ and $\rho_{n}$ by

$$
\Delta_{n}=\left\{\begin{array}{lll}
\left(1-1 / p_{n}, 1-1 / p_{n+1}\right), & \text { if } & p_{n+1}<2 p_{n}, \\
\left(1-1 / p_{n}, 1-1 / 2 p_{n}\right), & \text { if } & p_{n+1} \geqq 2 p_{n},
\end{array}\right.
$$

and $\rho_{n}=1-1 /\left(p_{n}+1\right)$ respectively.

(3.2) is obvious from (3.1) by Kronecker's theorem.

REMARK. The formula

$$
1 / d(x) \sim c p^{\prime}(x) / p(x)
$$

gives the relation between the strong summability factor $d(x)$ and the lacunary sequence $p(x)$. If we put $d(x)=1$, then $p(x)=2^{x}$, which is nothing but the Kolmogoroff theorem [2]. If we put $d(x)=x$, $x^{1 / 2}$, and so on we get $p(x)=x^{c}, 2^{c x^{1 / 2}}$, and so on, respectively.

We conclude with the following theorem which is proved by an argument due to Zygmund [7, 8, 9].

THEOREM 4. If $f(\theta)$ belongs to $L^{r}(r>1)$, then for almost all $\theta$, the sequence $\{1,2, \cdots\}$ can be divided into two complementary subsequences $\left\{n_{k}\right\}$ and $\left\{m_{k}\right\}$, depending in general on $\theta$, and such that

$$
s_{p_{n_{k}}}(\theta) \text { tends to } f(\theta) \text {, }
$$

the series $\sum 1 / d_{m *}$ converges,

where $d_{n} \uparrow, \sum 1 / d_{n}=\infty$, and

$$
p_{n} / d_{n}=O\left(p_{n+1}-p_{n}\right) .
$$

\section{BIBLIOGRAPHY}

1. R. P. Boas, Jr., and S. Bochner, On d theorem of M. Riesz for Fourier series, J. London Math. Soc. vol. 14 (1939) pp. 62-73. 
2. A. Kolmogoroff, Une contribution de l'étude de la convergence des series de Fourier, Fund. Math. vol. 5 (1924) pp. 96-97.

3. J. E. Littlewood and R. E. A. C. Paley, Theorems on Fourier series and power series, J. London Math. Soc. vol. 6 (1931) pp. 230-233.

4. - Theorems on Fourier series and power series, (2), Proc. London Math. Soc. (2) vol. 42 (1937) pp. 52-89.

5. J. Marcinkiewicz and A. Zygmund, $A$ theorem of Lusin, Duke Math. J. vol. 4 (1938) pp. 473-485.

6. G. Sunouchi, On the strong summability of Fourier series, Proc. Imp. Acad. Tokyo vol. 19 (1943) pp. 420-423.

7. Z. Zalcwasser, Sur la sommabilité des séries de Fourier, Studia Mathematica vol. 6 (1936) pp. 82-88.

8. A. Zygmund, Sur la caractère de divergence des séries orthogonales, Mathematica vol. 9 (1935) pp. 86-88.

9. - Proof of a theorem of Paley, Proc. Cambridge Philos. Soc. vol. 34 (1938) pp. 125-133.

10. - On the convergence and summability of power series on the circle of convergence (1), Fund. Math. vol. 30 (1938) pp. 170-196.

TOHOKU IMPERIAL UNIVERSITY

\section{ON THEOREMS OF M. RIESZ AND ZYGMUND}

\section{A. P. CALDERón}

Several proofs have been given of the results of M. Riesz and Zygmund.

(a) The conjugate of the Fourier series of a function $f(x)$ of $L^{p}, p>1$, is Fourier series of a function $\bar{f}(x)$ of the same class, and

$$
\int_{0}^{2 \pi}|\bar{f}(x)|^{p} d x \leqq A_{p} \int_{0}^{2 \pi}|f(x)|^{p} d x
$$

holds, $A_{p}$ is a constant depending only on $p$.

(b) If the function $|f(x)| \log ^{+}|f(x)|$ is integrable, the conjugate of the Fourier series of $f(x)$ is the Fourier series of a function $\bar{f}(x)$ of the class $L$. Moreover, there exist two constants $A$ and $B$ such that

$$
\int_{0}^{2 \pi}|\bar{f}(x)| d x \leqq A \int_{0}^{2 \pi}|f(x)| \log ^{+}|f(x)| d x+B .
$$

In view of the importance of these theorems it may be of interest to give another proof of them based on a different idea. Actually it is

\footnotetext{
Received by the editors May 14, 1949.
} 